\title{
Musculoskeletal pain and Menopause
}

Dr Fiona Watt

University Research Lecturer, Kennedy Institute of Rheumatology, University of Oxford, UK Honorary Consultant Rheumatologist, Nuffield Orthopedic Centre, Oxford, UK

Fiona E. Watt (M.D, Ph.D) ${ }^{\dagger}$

Arthritis Research UK Centre for Osteoarthritis Pathogenesis

Kennedy Institute of Rheumatology

Nuffield Department of Orthopaedics, Rheumatology and Musculoskeletal Sciences

University of Oxford

Oxford, OX3 7FY, United Kingdom.

${ }^{+}$Corresponding author and to whom reprint requests should be addressed: Dr Fiona Watt

Tel. +44 (0) 1865612600

Fax. +44 (0) 1865612621

Email: fiona.watt@kennedy.ox.ac.uk

Running title: MSK pain and Menopause

Key Words: menopause, estrogen, hormone, arthralgia, osteoarthritis, rheumatoid arthritis, management 


\begin{abstract}
Musculoskeletal pain, arthralgia and arthritis are all more common in women, and their frequency increases with age and in some appears to be associated with the onset of menopause. The clinical assessment, investigation and management of women presenting with musculoskeletal pain, arthralgia or arthritis at the time of menopause is reviewed. Common causes of arthralgia and arthritis in this population are discussed. The epidemiological and trials evidence for the effects of hormone replacement therapy (HRT) on musculoskeletal pain and arthritis (primarily from RCTs of HRT for other menopausal symptoms) are discussed. Lastly the possible underlying aetiological roles of sex hormones including estrogen, and their deficiency, in predisposing to musculoskeletal pain and arthritis are overviewed. Although the association appears strong, a causal link between estrogen deficiency and musculoskeletal pain or different types of arthritis is lacking; there have been few studies specifically within this group of symptomatic patients, and there is much still to understand about musculoskeletal pain and arthritis at the time of the menopause, and about how we might prevent or treat this.
\end{abstract}




\section{Introduction}

Chronic musculoskeletal pain represents an enormous health burden, affecting over $50 \%$ of adult populations. Common sites of pain are the spine, knee, hand, hip and shoulder ${ }^{1,2}$. There is a female preponderance irrespective of the cause of the pain $^{1,3,4}$. At the time of menopause, musculoskeletal pain is reported by more than half of women ${ }^{5}$. Presentation with joint pain in women is greatest between 45 and 55 years of age $e^{6}$. Although this appears to implicate the menopause and estrogen deficiency, direct causal evidence of a role for menopause is lacking, partly because musculoskeletal pain is so common throughout life. Women are approximately twice as likely to have joint pain and stiffness around the time or after the menopause than their premenopausal counterparts, when adjusted for age ${ }^{5}, 7$. However, many such epidemiological studies do not differentiate musculoskeletal pain from arthritis, so assessing the burden of arthralgia and arthritis in these populations is difficult.

Arthralgia is a subjective term, describing pain in a joint or joints. The implication is that this does not cause harm to the joints, and is quite separate from arthritis, which refers to an underlying pathological process within the joint associated with clinical symptoms but also signs. It is imperative to consider early arthritis, or another underlying inflammatory rheumatic disorder in anyone presenting with joint pain. However patients experiencing arthralgia around the time of menopause often do not have or develop an associated arthritis. The pain may be associated with hormonal changes at this time, or other secondary reversible conditions which are important to exclude ${ }^{8}$. In this review, the clinical approach to the peri- or post-menopausal woman with joint pain will be set out, including investigations and management. Possible pathogenetic factors in the development of musculoskeletal pain in relation to the menopause will be discussed, the evidence for the effects of hormone replacement therapy (HRT) in this setting and areas with lack of evidence will be highlighted.

\section{Clinical assessment of the menopausal patient with joint pain}

A careful history is essential when this symptom is first volunteered. Musculoskeletal pain does not always emanate from the joint, and should therefore not always be referred to as arthralgia: there are many different sources of musculoskeletal pain (Table 1). Pain may emanate from bone, muscles, bursae or entheses (points of insertion into bone of ligaments or tendons) away from the site of the joint. For example, rotator cuff tendinopathy is a common cause of shoulder pain in this age group. Objective measures e.g. pain visual analogue scale or numerical rating scale from 0-10 for average or worst joint pain should be used. Prolonged early morning stiffness (daily, greater than 30 minutes, or present with inactivity) is highly suggestive of an inflammatory cause, although can be 
present in arthralgia or osteoarthritis too. Where pain is non-specific, felt away from joints and in all four quadrants, fibromyalgia may be present. Red flags, such as weight loss, fevers or night pain should be documented and investigated urgently, excluding polymyalgia rheumatica (the commonest form of vasculitis, more common over the age of 50), neoplasia or atypical infection (Table 1).

A history of joint injury or occupational over-use, relevant preceding conditions (such as rash or flulike symptoms suggest viral aetiology), past or family history of psoriasis, uveitis or inflammatory bowel disease (increasing likelihood of inflammatory arthritis) and history of menopause and associated symptoms should be sought. A history of symptoms suggesting secondary causes of arthralgia and myalgia in this group include metabolic conditions such as thyroid disease or vitamin D deficiency should be sought (Table 2).

For the non-specialist, it is important to talk, look, feel and ask the patient to move painful joints, documenting the 3 ' $\mathrm{S}$ 's. This will often differentiate between arthralgia, pre-arthritis, or different types of arthritis (Table 3).

\section{Investigation of the patient with polyarthralgia or arthritis}

Further blood testing may often be helpful in diagnosing inflammatory arthritis or secondary causes where indicated. Useful tests are listed in Table 4. These should only be used to supplement clinical assessment, and not in place of it. Imaging may not be routinely required to make a diagnosis: in certain circumstances, plain radiography or musculoskeletal ultrasound may be useful to confirm diagnosis, stage the disease or assess prognosis. 


\section{General management}

If arthritis is suspected, it is important that a likely diagnosis of the type is made, as management and treatment options vary widely. Patients with features of rheumatoid arthritis should be referred immediately for review by a rheumatologist in an early arthritis clinic, Table $5^{9}$. If a secondary cause(s) or exacerbating factor is found, this should be treated and symptoms reviewed. It can take months for arthralgia from vitamin D deficiency or hypothyroidism to fully resolve; however, corticosteroid treatment of polymyalgia rheumatica typically improves pains within 48 hours.

In the case of arthralgia, the likely cause should be identified as far as possible (Table 2) and management with the aim of improving pain instituted. Identification of a possible drug cause for arthralgia needs careful discussion between the patient, physician and other relevant health care professionals, taking into account the effects on quality of life, and the relative risks and benefits of staying on the drug versus reducing drug dose, switching to another drug within the same or different class or simple cessation. Statin-induced myalgia or arthralgia is actually extremely uncommon (other causes of pain should be actively sought); if suspected on temporal grounds, the risk-benefit of reducing or stopping the drug should be carefully discussed and documented, prior to either drug holiday, cessation or class-switching.

\section{Common forms of arthralgia occurring in perimenopause}

\section{Menopause-associated arthralgia}

Arthralgia is a menopausal symptom in at least $50 \%$ of women (musculoskeletal pain is one of the most commonly reported symptoms in international populations $)^{10-13}$. In this situation, by definition no secondary cause has been found.

\section{Clinical features}

Usually pain is not the main symptom but part of a wider syndrome. However, it is the predominant symptom in $21 \%$ of women ${ }^{5}$. Arthralgia and muscle pain of any origin is often associated with fatigue, mood change, sleep disturbance, raised body mass index (BMI), anxiety or stress ${ }^{14}$. Given that these features are also frequently also associated with menopause, they can have both a potentiating effect on the presence of arthralgia, and be compounded by its presence. Menopauseassociated arthralgia is typically transient and self-limiting and may not present to a physician, but may be a source of persistent pain for a minority of women, or evolve into arthritis or chronic widespread pain in susceptible individuals. For this reason, persisting arthralgia should be intermittently reviewed to ensure no change in the diagnosis. 


\section{Management}

There is little evidence base for the management of menopause-associated arthralgia. General advice for musculoskeletal pain is to control weight (with planned weight loss where BMI is raised) and to take regular exercise. This can be both aerobic and joint-specific. There is some evidence that moderate levels of physical activity improves peri-menopausal arthralgia and other menopauserelated symptoms ${ }^{15}$. If there is deconditioning evident, a graded and gradual increase in exercise should be planned, avoiding sudden overloading of painful joints, which may exacerbate pain and cause loss of confidence and adherence. Avoidance of heavy lifting or repetitive joint use, for example in hand arthralgia, is often helpful.

Supportive treatment should also aim to improve symptoms of stress, anxiety or depression and sleep quality (if sleep disturbance is present). Again, there is little or no trial evidence in this setting, although good evidence in related conditions such as fibromyalgia ${ }^{16}$. Dietary measures aiming to regulate estrogen levels may help improve arthralgia. A recent controlled clinical trial showed that a complex intervention which included dietary and exercise supervision reduced arthralgia ${ }^{17}$. The use of supplements such as phytoestrogens, evening primrose oil, starflower oil, fish oils or ginseng are reportedly used by $32 \%$ of post-menopausal women for non-vasomotor symptoms including arthralgia $^{18}$. These agents may be helpful in controlling menopausal symptoms but a lack of evidence for their effects specifically on arthralgia.

Where pain is persistent and moderate to severe, a regular analgesic such as acetaminophen may be required. It should only be continued if found to be effective. Non-steroidal anti-inflammatory drugs (NSAIDs) may be helpful either topically or orally in managing persistent symptoms unresponsive to acetaminophen, although there is a lack of evidence for this specific setting. Sparing use and monitoring of oral agents is advisable because of potential gastrointestinal, renal or cardiovascular toxicity.

Current indications for systemic HRT are vasomotor and gynecological menopausal symptoms not responding to local or conservative therapies. Joint pain and stiffness has been noticed to be modestly reduced in the active arms of RCTs including WHI $(76.3 \% \text { vs. } 79.2 \%, P=0.001)^{19}$ and an Australian $\mathrm{RCT}^{20}$. Epidemiological studies in this area are conflicting, as many patients may seek HRT because of musculoskeletal symptoms ${ }^{21}$. If arthralgia is severe and persisting, unresponsive to other measures, temporally related to menopause, where quality of life is impaired and particularly in the presence of other incapacitating menopausal symptoms, it may be reasonable to discuss whether HRT could be tried. Patient reported measures of pain and menopause symptoms and their change should be recorded. 


\section{Aromatase-induced Arthralgia}

Aromatase inhibitors used in the management of breast cancer in this group are a particularly common cause of arthralgia, and may be seen in as many as half of users ${ }^{22}$. If medically required and where there are no reasonable alternatives, active management of arthralgia symptoms may be necessary to maintain therapy ${ }^{23}$. Mild arthralgia should be treated symptomatically with lifestyle advice including exercise and weight loss, and response documented. For more severe symptoms, treatment with an oral analgesic such as acetaminophen or NSAID should be offered, to enable continuation of therapy (risk-benefit of NSAIDs/coxibs should be carefully considered). Any decision to modulate, switch or change a drug should always made by the treating oncologist. Switching to an alternative agent within- or between-class may sometimes lead to an improvement in symptoms. There is no clinical trial evidence to support the use of omega-3 oils to treat this type of arthralgia ${ }^{24}$.

\section{Fibromyalgia (Chronic Widespread Pain syndrome)}

Among the most common sources of chronic arthralgia and myalgia in adult women is fibromyalgia (previously known as myofascial pain syndrome). The cause of this condition is not fully understood: but genetic and hormonal influences are likely to be important in generating heightened pain sensitivity, and tendency to other chronic pain syndromes ${ }^{16,25}$.

\section{Clinical features}

Pain is usually present in all 4 quadrants: primarily axial, with diffuse aching in the neck, shoulders, back, and pelvis. A history of poor sleep (sleep disturbance, or seemingly adequate sleep but feeling unrefreshed in the morning) and persistent unexplained fatigue are typical. Arthralgia and myalgia is typically accompanied by multiple other symptoms, including tingling, burning or swelling of limbs. Female gender, catastrophizing personality, high depression or anxiety scores are all risk factors. There is no detectable joint swelling or synovitis (although peripheral puffiness may be seen) and muscle strength is normal. Characteristic tender 'trigger points' may be found, although these are not necessary for a diagnosis of Chronic Widespread Pain. Patients with fibromyalgia tend to display heightened pain sensitivity at other non-joint, non-spinal sites as well, or have pain affecting other systems, for example migraine, irritable bowel or bladder, premenstrual syndrome or pelvic pain, non-specific chest pain ${ }^{26}$. It is likely that there is a common pathogenesis to different manifestations of chronic pain. Many of these conditions, such as migraine, are more common at the time of menopause. The relationship of fibromyalgic symptoms to menopausal symptoms should be noted.

\section{Management}

Fibromyalgia is also a diagnosis of exclusion. Following appropriate clinical assessment and investigation, giving a clear diagnosis and full explanation is essential, to prevent ongoing, fruitless 
medical investigation and over-medicalisation. Advice on pacing, sleep hygiene, weight control and exercise are important ${ }^{16}$. Institution of an aerobic conditioning program with graded exercise can be helpful. Analgesia is often important, starting with standard analgesics such as acetaminophen, or NDAIDs where indicated. Opiates are often ineffective. Consistent with the concept that the pain of fibromyalgia is related to aberrant neurotransmission or pain processing, other neuroactive 'neuropathic' medications which have been approved for treatment of fibromyalgia include pregabalin, gabapentin, duloxetine and milnacipran. Tricyclic antidepressants such as low dose amitriptyline as well as the later-generation SSRIs are routinely used, the latter particularly when depression is present. Treatment of fibromyalgia may be challenging. Addressing any identifiable biopsychosocial aspects to the presentation and ongoing pain is essential. Some cases may benefit from multidisciplinary specialist pain management.

\section{Common forms of arthritis occurring in the peri-menopause}

\section{Osteoarthritis}

Osteoarthritis (OA) is the most common form of arthritis; the lifetime risk for women is $47 \%{ }^{27}$. It is a whole organ disease (affecting articular cartilage, bone, synovium, ligament) ${ }^{28}$. In many it leads to progressive loss of cartilage and change in bone (leading to classical 'osteophytes' on X-ray) with associated joint pain, swelling and loss of function (Table 3). OA ultimately leads to joint failure for many, and is the commonest reason for total joint replacement, with high associated socioeconomic $\operatorname{cost}^{29}$. However, in early disease, symptoms may often stabilise or improve, with marked variation in symptoms between individuals at any radiographic stage ${ }^{30}$.

Although the initiating processes are not fully understood, it is clear that osteoarthritis is an active, cellularly-driven process which includes excessive degradation and abnormal repair responses in joint tissues, in part driven by mechanical factors ${ }^{31}$. Blockade of critical pathways such as proteinases protects from the disease ${ }^{32}$; interestingly female mice are relatively protected, but this protection is lost following ovariectomy, implicating sex hormones in disease pathogenesis ${ }^{32}$. Important risk factors for OA include age, obesity, female gender, congenital joint abnormality or deformity, a history of substantial joint injury, excessive joint loading and family history ( $60 \%$ of risk is genetic) $)^{33}$.

\section{Clinical features}

The commonest affected sites are the knee, hip and hand. Pain affected joints can be intermittent or constant, typically worse with use (Table 3). In the hand, the distal interphalangeal and proximal interphalangeal joints are most commonly affected, followed by the base of the thumb. There may 
be apparent bony swelling ('nodes') of the interphalangeal joints, which if present are highly suggestive of OA. Blood tests listed in Table 4 are typically normal, although there may be a modestly raised CRP. Diagnostic changes are detectable by X-ray in established disease, but may be absent in early disease. At all sites, there is a female preponderance. Hand OA appears different to other forms in having a definite upsurge in incidence around the age of the menopause in women ${ }^{6}$. $\sim 90 \%$ of individuals in secondary care clinics with hand OA are female ${ }^{34,35}$. In such 'peri-menopausal' cases, symptoms may resolve over two to five years; however there is no proven causal link between estrogen deficiency and OA.

\section{Management}

A clear diagnosis of osteoarthritis should be made and shared with the patient. Management should be focused on the individual, including an assessment of the effect of OA on their work, leisure and quality of life. Patients should be educated and given constructive messages about their disease, including its natural history and possibility of improvement early in disease with or without intervention ${ }^{36,37}$. All patients with OA should be encouraged to remain in work and to exercise ${ }^{38}$. Management of osteoarthritis falls into four broad areas: supportive/lifestyle measures (encouraging weight control or loss, general exercise), non-pharmacological (such as joint-specific exercises, or devices such as base of thumb splints, or knee braces that mechanically 'offload' joints), pharmacological measures and ultimately evidence-based surgical interventions, such as joint replacement (there is no evidence that simple washout of the joint is helpful for those with OA, and this should be avoided $)^{39}$. Not all those with OA will need regular oral analgesia. For those who do, acetaminophen and topical NSAIDs are the first-line treatments. Stronger analgesia such as opiates or oral NSAIDs may be indicated, but should be used with caution given the risks associated with these drugs; intra-articular steroid injections are often helpful in managing symptoms and flares. The current incomplete understanding of this disease means that, unlike in rheumatoid arthritis, diseasemodifying drugs do not yet exist for OA. Newer agents which target pain-generating, or diseasemodifying biological pathways are currently in clinical trials.

There is at present no definite evidence for the use of HRT in this setting. Those in the estrogencontaining arm of WHI were noted on post-hoc testing to have significantly lower rates of joint arthroplasty (hip, and to a lesser extent knee) ${ }^{40}$. A protective effect of HRT on hand and knee radiographic disease has been described previously at a population level ${ }^{41}$. However, no interventional studies of HRT have been run specifically studying those with symptomatic osteoarthritis. 


\section{Rheumatoid arthritis}

Rheumatoid arthritis (RA) is a classical form of inflammatory arthritis. It is three times more common in women than men; peak age of onset is between ages 35 and 55, and it commonly affects perimenopausal and post-menopausal women ${ }^{42,43}$. Genetic factors, the possession of the HLA DR 'shared epitope', cyclic citrullinated peptide (CCP) antibodies (which may predate the disease by many years) and cigarette smoking all predispose to the disease ${ }^{44,45}$. RA often goes into remission during pregnancy, but despite this, a causal relationship for hormones has not yet been defined. HRT may reduce CCP antibodies in the presence of the shared epitope ${ }^{46}$ ). Synovial inflammation leads to 'pannus', a destructive, dysregulated tissue within the joint where infiltrating immune cells (lymphocytes and macrophages) release proteinases and pro-inflammatory cytokines such as tumor necrosis factor (TNF)- $\alpha$, leading to loss of articular cartilage and bony erosion ${ }^{47}$. Untreated, this leads to progressive, irreversible joint damage, with ensuing functional impairment and disability.

\section{Clinical features}

RA typically affects multiple peripheral joints in a symmetric and progressive manner, with associated signs of inflammation: pain, swelling, warmth, loss of function and sometimes redness (although marked erythema over joints is more typical in gout or septic arthritis) (Table 3). Prolonged morning stiffness is typically present in active disease, along with some systemic symptoms (fatigue, malaise, sweats) ${ }^{42}$. This systemic disease can involve other organs (lungs, pericardium, eyes, skin, nerve). Laboratory studies usually reveal elevated acute-phase proteins (ESR, CRP), Table 4; rheumatoid factor is commonly found in patients with RA. The possession of rheumatoid factor, or more specific anti-CCP, are associated with increased risk of erosion and joint damage, which is worse in smokers and those with shared epitope ${ }^{48}$. Ultrasound and other imaging modalities are used in the assessment and monitoring of RA ${ }^{49}$.

\section{Management}

The treatment of RA has been transformed in the last 20 years by the new biologics era. Early diagnosis (ahead of damage) is vital, and rapid, early disease modification, initially with diseasemodifying anti-rheumatic drugs (DMARDs). Treatment is escalated until disease remission is achieved, with use of biologic therapies such as anti-TNF where necessary ${ }^{42,50,51}$. A detailed review of therapeutics in this area is outside of scope.

What effect does HRT have on RA? In small RCTs, it appears safe (checking usual exclusions: cardiovascular risk is increased in those with RA, so this should be borne in mind when prescribing). No significant effect on incidence or severity of RA was seen in the active ams of WHI ${ }^{52}$. HRT has been reported to mitigate against bone loss and improve some symptoms ${ }^{46,53}$. 


\section{Aetiology: sex hormones, musculoskeletal pain and inflammation}

Cartilage. Estrogen is synthesised by aromatase in connective tissues and estrogen receptors are present in all joint tissues including articular cartilage, subchondral bone, ligaments and synovium ${ }^{54}$. Low level estrogens appear to promote cartilage growth or prevent its degradation ${ }^{55,56}$. After ovary removal, resistance to articular cartilage compressibility is reduced, but appears to be reversed by estrogen replacement ${ }^{57}$. Increased articular cartilage is seen by MRI in those taking HRT ${ }^{58}$. Many animal studies suggest estrogen and selective estrogen receptor modulators (SERMs) may be protective of arthritis ${ }^{54,59}$. However, there is far less data on the effects of HRT on musculoskeletal pain and arthritis in humans.

Inflammation: Estrogen is known to be anti-inflammatory and mildly immunosuppressive. Its role as a steroid hormone of pregnancy requires this. (Younger) rheumatoid arthritis patients routinely note improvement or even remission during pregnancy, and much of this effect is attributed to the antiinflammatory properties of estrogen. Joint damage in arthritis is driven by activation of inflammatory signalling pathways inducing proteinases ${ }^{28}$. HRT and SERMs such as levormeloxifene and raloxifene have been reported to reduce levels of inflammation and markers of subsequent matrix degradation ${ }^{60-62}$.

Bone: The effects of estrogen on bone turnover are well known from osteoporosis, although other therapeutic groups have superseded HRT in its treatment. Bone-targetting agents would appear beneficial in some forms of arthritis too ${ }^{62}$. In a cross-sectional study, women receiving estrogen had less OA-related bony changes on knee MRI than those who were not ${ }^{63}$. In monkeys, estrogen replacement after ovariectomy appeared to limit new bone formation seen in $\mathrm{OA}^{64}$.

Pain: Estrogen receptors and aromatase are present in dorsal root ganglion, hypothalamus, limbic system, neurone and joint. Estrogen therapy has been shown to decrease synovial nerve fibre neurotophins in an animal model of $\mathrm{OA}^{65}$. Estrogen and testosterone both reduce pain, activating inhibitory pain pathways in the spinal cord, whilst progestins have been reported to promote pain ${ }^{66}$. During normal menstrual cycle, drops in estrogen were associated with increased pain reporting using objective measures of pain induction, whereas high estrogen/low progestin was associated with activation of the endogenous opioid system, and less pain ${ }^{67,68}$. Those with low testosterone states appear more prone to pain ${ }^{68}$. Entering menopause may for some be like constantly being in the 'low estrogen' part of the cycle, heightening the pain experience for any given pathology. Fatigue, poor sleep and mood change occurring in menopause are also well known to enhance pain perception. 


\section{Conclusions}

Musculoskeletal pain is more common in women, and increases during and after menopause. Not all musculoskeletal pain is arthralgia, and not all joint pain is arthritis. Around the menopause, women have increased prevalence of OA (particularly hand) and RA. Those with symptoms and signs suggesting inflammatory arthritis such as rheumatoid arthritis should be referred urgently to an early arthritis clinic for further assessment. Definitive evidence for menopausal hormonal treatment in the prevention or treatment of either form of arthritis is lacking. There is some limited evidence HRT may help musculoskeletal symptoms or OA at a population level, but currently insufficient evidence to recommend it on an individual basis. If onset of arthralgia, or arthritis is near to the menopause and other related symptoms, it is reasonable to treat possible reversible causes and other systemic menopausal symptoms and record the response of musculoskeletal symptoms to these treatments. However, there have been few studies and no RCTs specifically including this group of symptomatic patients. There is much still to understand about musculoskeletal pain and arthritis at the time of the menopause, and about how we might prevent or treat this.

\section{Practice Points}

- In a patient with musculoskeletal pain, be clear on the source of the pain and likely diagnosis

- Not all arthralgia evolves in to arthritis, but some may

- Menopause-associated arthralgia is a diagnosis of exclusion: consider other potential secondary causes

- The commonest form of arthritis is osteoarthritis; hand osteoarthritis onset is more frequent around the time of the menopause

- Suspected inflammatory arthritis such as rheumatoid arthritis should be urgently referred to a rheumatologist for management

- Lifestyle measures (weight control, dietary measures, exercise and sleep hygiene) are first line interventions for arthralgia or osteoarthritis

- There is a lack of current evidence to use HRT specifically to treat arthralgia, fibromyalgia, osteoarthritis or rheumatoid arthritis

- HRT may have a role when musculoskeletal symptoms are part of a wider syndrome of menopause which has not responded to conservative measures 
Table 1 Causes of musculoskeletal pain in the perimenopause

\begin{tabular}{|c|c|}
\hline Joint-based pain (arthralgia) & $\begin{array}{l}\text { Primary/Idiopathic arthralgia } \\
\text { (Menopausal-associated arthralgia) } \\
\text { Arthralgia due to secondary causes (see } \\
\text { Table 2) } \\
\text { Arthritis (osteoarthritis, inflammatory } \\
\text { arthritis e.g. rheumatoid arthritis, } \\
\text { psoriatic arthritis, gout, pseudogout). }\end{array}$ \\
\hline Myalgia & $\begin{array}{l}\text { Endocrine (Vitamin D, deficiency, thyroid, } \\
\text { Cushing's) } \\
\text { Menopausal } \\
\text { Polymyalgia rheumatic } \\
\text { Drug induced (statins, fibrates) }\end{array}$ \\
\hline Entheseal/tendon/bursitis pain & $\begin{array}{l}\text { Injury } \\
\text { Asymmetric gait/overload } \\
\text { (obesity/biomechanical factors such as } \\
\text { flat feet) } \\
\text { Drug-induced (quinolones) }\end{array}$ \\
\hline Bone pain & $\begin{array}{l}\text { Metabolic (Paget's disease) } \\
\text { Neoplasia (Myeloma, metastatic disease) } \\
\text { Infection (Brucellosis, Tuberculuosis) } \\
\text { Fracture (Trauma, Osteoporotic fracture) }\end{array}$ \\
\hline Fibromyalgia & $\begin{array}{l}\text { Associated with: Fatigue, Sleep } \\
\text { disturbance, anxiety or depression, } \\
\text { catastrophizing, other pain syndromes } \\
\text { e.g. migraine, IBS, atypical chest pain }\end{array}$ \\
\hline
\end{tabular}


Table 2 Causes of arthralgia in menopausal women

\begin{tabular}{|c|c|}
\hline Cause & Features \\
\hline Primary/idiopathic & $\begin{array}{l}\text { Timing (and presence) of other estrogen } \\
\text { deficiency symptoms (absence of } \\
\text { identifiable secondary causes) }\end{array}$ \\
\hline \multicolumn{2}{|c|}{ Secondary causes } \\
\hline $\begin{array}{l}\text { Endocrine } \\
\text { - Hypothyroidism } \\
\text { - Hyperparathyroidism (primary or secondary) } \\
\text { - Vitamin D deficiency } \\
\text { - Anaemia }\end{array}$ & $\begin{array}{l}\text { Fatigue; weight gain; hyporeflexia; } \\
\text { proximal myopathy } \\
\text { Abdominal pains; high calcium } \\
\text { Fatigue; proximal myopathy } \\
\text { Fatigue; shortness of breath }\end{array}$ \\
\hline $\begin{array}{l}\text { Drug-related } \\
\text { - Statins and other lipid-lowering drugs } \\
\text { - Aromatase inhibitors } \\
\text { - Selective Estrogen Receptor Modulators (SERMs) } \\
\text { - Bisphosphonates (particularly intravenous) } \\
\text { - Thiazide diuretics }\end{array}$ & $\begin{array}{l}\text { Relevant temporal history } \\
\text { Response, if appropriate, to drug } \\
\text { holiday/cessation (aromatase inhibitors } \\
\text { should not be stopped without } \\
\text { oncology guidance) }\end{array}$ \\
\hline $\begin{array}{l}\text { Metabolic } \\
\text { - Liver disease } \\
\text { - } \text { Renal disease }\end{array}$ & $\begin{array}{l}\text { Appropriate history, or abnormality on } \\
\text { blood testing }\end{array}$ \\
\hline $\begin{array}{l}\text { Rheumatic } \\
\text { - Connective tissue disease (lupus*, scleroderma, } \\
\text { Sjogrens*) } \\
\text { - Sarcoidosis* } \\
\text { - Vasculitis } \\
\text { - Hyperuricaemia } \\
\text { - Hypermobility }\end{array}$ & $\begin{array}{l}\text { Rashes, oral ulcers, other clinical } \\
\text { features of the disease } \\
\text { Other blood test abnormalities, e.g. } \\
\text { ANA or ANCA positivity, raised serum } \\
\text { ACE, raised serum urate } \\
\text { Evidence of hypermobility on } \\
\text { examination }\end{array}$ \\
\hline $\begin{array}{l}\text { Infection } \\
\text { - Parvovirus* } \\
\text { - Hepatitis B*/C*/HIV* } \\
\text { - Ross River Virus* } \\
\text { - Brucellosis* } \\
\text { - Whipple's disease* } \\
\text { - Lyme disease* }\end{array}$ & $\begin{array}{l}\text { Relevant rash, viral symptoms } \\
\text { Relevant travel or other risk history } \\
\text { History of insect bite }\end{array}$ \\
\hline $\begin{array}{l}\text { Malignancy } \\
\text { - Disseminated bony malignancy } \\
\text { - Paraneoplastic syndrome }\end{array}$ & $\begin{array}{l}\text { Red flags e.g. weight loss, bone pain, } \\
\text { fever } \\
\text { Other clinical features of malignancy }\end{array}$ \\
\hline
\end{tabular}

* May be associated with arthralgia or a frank arthritis 
Table 3 Differentiating features of arthritis: the ' $3 \mathrm{~S}$ ' approach

\begin{tabular}{|c|c|c|c|}
\hline & RA* & $\mathrm{OA}$ & Gout/pseudogout* \\
\hline \multicolumn{2}{|l|}{ 1. Does the patient have joint-based site? $Y$} & $\mathrm{Y}$ & $\mathrm{Y}$ \\
\hline - Single, or multiple? & Multiple & Either & Typically single or few \\
\hline - Symmetrical? & Y & $\mathrm{Y} / \mathrm{N}$ & $\mathrm{N}$ \\
\hline - Episodic vs. progressive? & Progressive* & Either & Episodict \\
\hline - With activity? & Variable & Y & Sometimes \\
\hline \multicolumn{2}{|c|}{ 2. Is there early morning stiffness $>30 \mathrm{~min}$ ? $\mathrm{Y}$} & $\mathrm{N}$ & Y (flares) \\
\hline 3. Can I see joint swelling? & $\mathrm{Y}$ & $\mathrm{Y}$ & $\mathrm{Y}$ \\
\hline - $\quad$ Are the joints red? & $\mathrm{N}$ & $\mathrm{N}$ & Y (flares) \\
\hline - $\quad$ Are the joints tenderł? & $\mathrm{Y}$ & $\mathrm{Y} / \mathrm{N}$ & Y (flares) \\
\hline - Is there MCP joint swelling? & Usually & Occasic & Occasionally \\
\hline
\end{tabular}

‡ Tenderness is usually present in arthritis, but not always in arthralgia

* RA Early, established disease (rarely palindromic RA can occur, with episodes of flare and remission.

†Gout. Based on new presentation of gout. Established, tophaceous gout can give persistent pain, and progressive arthritis. 
Table 4. Laboratory investigations for polyarthralgia and arthritis

Full blood count \& biochemistry profile including urea and electrolytes, liver function tests, calcium

Acute phase proteins: CRP, ESR

Serum Urate

Creatine kinase

Thyroid function, Parathyroid hormone

Iron studies, B12, folate (if anaemia suspected)

Vitamin D

Autoantibodies* (Antinuclear antibody, rheumatoid factor, cyclic citrullinated peptide (CCP) antibodies)

Viral or other serological testing e.g. Parvovirus B19, Hepatitis B, C, HIV (where indicated)

*Autoantibodies may be positive in the absence of inflammatory arthritis. Only request these tests in the context of symptoms and signs of inflammatory arthritis. 
Table 5 Features suggesting rheumatoid arthritis

-Symptoms persisting more than 2 weeks

- Swollen joints especially in hands (wrists, metacarpophalangeal joints, proximal interphalangeal joints)

-Tenderness across the metatarsophalangeal joints

- Positive rheumatoid factor

-Positive anti-citrullinated protein antibodies

-Elevated inflammatory markers (erythrocyte sedimentation rate, C-reactive protein)

-Number of swollen joints

-Symmetrical pattern (can start asymmetrically)

-Absence of an alternative diagnosis such as erosive osteoarthritis, crystal arthritis

Reproduced with permission (NB need to seek permission) from ${ }^{9}$. 


\section{Competing interest statement}

FW has received research grants supporting clinical studies from Astellas Pharma Inc. and Pfizer Inc.

\section{Acknowledgements}

This work was supported by the Arthritis Research UK Centre for Osteoarthritis

Pathogenesis, grant ref. 20205 and by the National Institute for Health Research (NIHR)

Oxford Biomedical Research Centre (BRC).

The views expressed are those of the author and not necessarily those of the NHS, the NIHR or the Department of Health. 


\section{References}

1. Urwin $\mathrm{M}$, Symmons $\mathrm{D}$, Allison $\mathrm{T}$, et al. Estimating the burden of musculoskeletal disorders in the community: the comparative prevalence of symptoms at different anatomical sites, and the relation to social deprivation. Annals of the rheumatic diseases. 1998; 57: 649-55.

2. Gao HL, Lin SQ, Wei $Y$, Chen $Y$ and Wu ZL. The effect of age and menopausal status on musculoskeletal symptoms in Chinese women aged 35-64 years. Climacteric : the journal of the International Menopause Society. 2013; 16: 639-45.

3. Fayaz A, Croft P, Langford RM, Donaldson $L$ and Jones GT. Prevalence of chronic pain in the UK: a systematic review and meta-analysis of population studies. BMJ open. 2016; 6: e010364.

4. Wijnhoven $\mathrm{HA}$, de Vet $\mathrm{HC}$ and Picavet HS. Explaining sex differences in chronic musculoskeletal pain in a general population. Pain. 2006; 124: 158-66.

5. Szoeke CE, Cicuttini FM, Guthrie JR and Dennerstein L. The relationship of reports of aches and joint pains to the menopausal transition: a longitudinal study. Climacteric : the journal of the International Menopause Society. 2008; 11: 55-62.

6. Prieto-Alhambra D, Judge A, Javaid MK, Cooper C, Diez-Perez A and Arden NK. Incidence and risk factors for clinically diagnosed knee, hip and hand osteoarthritis: influences of age, gender and osteoarthritis affecting other joints. Annals of the rheumatic diseases. 2014; 73: 1659-64.

7. Ho SC, Chan SG, Yip YB, Cheng A, Yi Q and Chan C. Menopausal symptoms and symptom clustering in Chinese women. Maturitas. 1999; 33: 219-27.

8. Magliano M. Menopausal arthralgia: Fact or fiction. Maturitas. 2010; 67: 29-33.

9. Aletaha D, Neogi T, Silman AJ, et al. 2010 Rheumatoid arthritis classification criteria: an American College of Rheumatology/European League Against Rheumatism collaborative initiative. Arthritis and rheumatism. 2010; 62: 2569-81.

10. Chim H, Tan BH, Ang CC, Chew EM, Chong YS and Saw SM. The prevalence of menopausal symptoms in a community in Singapore. Maturitas. 2002; 41: 275-82.

11. Obermeyer CM, Reher D, Alcala LC and Price K. The menopause in Spain: results of the DAMES (Decisions At MEnopause) study. Maturitas. 2005; 52: 190-8.

12. Olaolorun FM and Lawoyin TO. Experience of menopausal symptoms by women in an urban community in Ibadan, Nigeria. Menopause. 2009; 16: 822-30.

13. Syed Alwi SA, Lee PY, Awi I, Mallik PS and Md Haizal MN. The menopausal experience among indigenous women of Sarawak, Malaysia. Climacteric : the journal of the International Menopause Society. 2009; 12: 548-56. 
14. Conboy L, Domar A and O'Connell E. Women at mid-life: symptoms, attitudes, and choices, an internet based survey. Maturitas. 2001; 38: 129-36.

15. Aparicio VA, Borges-Cosic M, Ruiz-Cabello $P$, et al. Association of objectively measured physical activity and physical fitness with menopause symptoms. The Flamenco Project. Climacteric : the journal of the International Menopause Society. 2017: 1-6.

16. Rahman A, Underwood M and Carnes D. Fibromyalgia. BMJ. 2014; 348: g1224.

17. Xi S, Mao L, Chen X and Bai W. Effect of health education combining diet and exercise supervision in Chinese women with perimenopausal symptoms: a randomized controlled trial. Climacteric : the journal of the International Menopause Society. 2017; 20: 151-6.

18. Gartoulla P, Davis SR, Worsley R and Bell RJ. Use of complementary and alternative medicines for menopausal symptoms in Australian women aged 40-65 years. The Medical journal of Australia. 2015; 203: 146, e 1-6.

19. Chlebowski RT, Cirillo DJ, Eaton CB, et al. Estrogen alone and joint symptoms in the Women's Health Initiative randomized trial. Menopause. 2013; 20: 600-8.

20. Welton AJ, Vickers MR, Kim J, et al. Health related quality of life after combined hormone replacement therapy: randomised controlled trial. BMJ. 2008; 337: a1190.

21. Watt FE. Hand osteoarthritis, menopause and menopausal hormone therapy. Maturitas. 2016; 83: 13-8.

22. Lintermans A and Neven P. Pharmacology of arthralgia with estrogen deprivation. Steroids. 2011; 76: 781-5.

23. Coleman RE, Bolten WW, Lansdown M, et al. Aromatase inhibitor-induced arthralgia: clinical experience and treatment recommendations. Cancer treatment reviews. 2008; 34: 275-82.

24. Hershman DL, Unger JM, Crew KD, et al. Randomized Multicenter Placebo-Controlled Trial of Omega-3 Fatty Acids for the Control of Aromatase Inhibitor-Induced Musculoskeletal Pain: SWOG S0927. Journal of clinical oncology : official journal of the American Society of Clinical Oncology. 2015; 33: 1910-7.

25. Livshits G, Macgregor AJ, Gieger C, et al. An omics investigation into chronic widespread musculoskeletal pain reveals epiandrosterone sulfate as a potential biomarker. Pain. 2015; 156: 1845-51.

26. Vincent A, Whipple MO, McAllister SJ, Aleman KM and St Sauver JL. A cross-sectional assessment of the prevalence of multiple chronic conditions and medication use in a sample of community-dwelling adults with fibromyalgia in Olmsted County, Minnesota. BMJ open. 2015; 5: e006681. 
27. Losina $E$, Weinstein $A M$, Reichmann $W M$, et al. Lifetime risk and age at diagnosis of symptomatic knee osteoarthritis in the US. Arthritis care \& research. 2013; 65: 703-11.

28. Bijlsma JW, Berenbaum F and Lafeber FP. Osteoarthritis: an update with relevance for clinical practice. Lancet. 2011; 377: 2115-26.

29. Losina $E$, Paltiel $A D$, Weinstein $A M$, et al. Lifetime medical costs of knee osteoarthritis management in the United States: impact of extending indications for total knee arthroplasty. Arthritis care \& research. 2015; 67: 203-15.

30. Nicholls E, Thomas E, van der Windt DA, Croft PR and Peat G. Pain trajectory groups in persons with, or at high risk of, knee osteoarthritis: findings from the Knee Clinical Assessment Study and the Osteoarthritis Initiative. Osteoarthritis and cartilage / OARS, Osteoarthritis Research Society. 2014; 22: 2041-50.

31. Glyn-Jones S, Palmer AJ, Agricola R, et al. Osteoarthritis. Lancet. 2015; 386: 376-87.

32. Ma HL, Blanchet TJ, Peluso D, Hopkins B, Morris EA and Glasson SS. Osteoarthritis severity is sex dependent in a surgical mouse model. Osteoarthritis and cartilage / OARS, Osteoarthritis Research Society. 2007; 15: 695-700.

33. Loughlin J. Genetic contribution to osteoarthritis development: current state of evidence. Current opinion in rheumatology. 2015; 27: 284-8.

34. Cecil RL and Archer BH. Arthritis of the menopause: A study of fifty cases. Journal of the American Medical Association. 1925; 84: 75-9.

35. Punzi L, Ramonda R and Sfriso P. Erosive osteoarthritis. Best practice \& research Clinical rheumatology. 2004; 18: 739-58.

36. Zhang W, Moskowitz RW, Nuki G, et al. OARSI recommendations for the management of hip and knee osteoarthritis, Part II: OARSI evidence-based, expert consensus guidelines. Osteoarthritis and cartilage / OARS, Osteoarthritis Research Society. 2008; 16: 137-62.

37. Zhang W, Nuki G, Moskowitz RW, et al. OARSI recommendations for the management of hip and knee osteoarthritis: part III: Changes in evidence following systematic cumulative update of research published through January 2009. Osteoarthritis and cartilage / OARS, Osteoarthritis Research Society. 2010; 18: 476-99.

38. Conaghan PG, Dickson J and Grant RL. Care and management of osteoarthritis in adults: summary of NICE guidance. BMJ. 2008; 336: 502-3.

39. Fernandes L, Hagen KB, Bijlsma JW, et al. EULAR recommendations for the nonpharmacological core management of hip and knee osteoarthritis. Annals of the rheumatic diseases. 2013; 72: 1125-35. 
40. Cirillo DJ, Wallace RB, Wu L and Yood RA. Effect of hormone therapy on risk of hip and knee joint replacement in the Women's Health Initiative. Arthritis Rheum. 2006; 54: 3194-204.

41. Spector TD, Nandra D, Hart DJ and Doyle DV. Is hormone replacement therapy protective for hand and knee osteoarthritis in women?: The Chingford Study. Annals of the rheumatic diseases. 1997; 56: 432-4.

42. Smolen JS, Aletaha D and McInnes IB. Rheumatoid arthritis. Lancet. 2016; 388: 2023-38.

43. Carmona L, Cross M, Williams B, Lassere M and March L. Rheumatoid arthritis. Best practice \& research Clinical rheumatology. 2010; 24: 733-45.

44. Potempa J, Mydel P and Koziel J. The case for periodontitis in the pathogenesis of rheumatoid arthritis. Nature reviews Rheumatology. 2017.

45. Mahdi $\mathrm{H}$, Fisher BA, Kallberg $\mathrm{H}$, et al. Specific interaction between genotype, smoking and autoimmunity to citrullinated alpha-enolase in the etiology of rheumatoid arthritis. Nature genetics. 2009; 41: 1319-24.

46. Salliot C, Bombardier C, Saraux A, Combe B and Dougados M. Hormonal replacement therapy may reduce the risk for RA in women with early arthritis who carry HLA-DRB1 *01 and/or *04 alleles by protecting against the production of anti-CCP: results from the ESPOIR cohort. Annals of the rheumatic diseases. 2010; 69: 1683-6.

47. Williams RO, Feldmann M and Maini RN. Cartilage destruction and bone erosion in arthritis: the role of tumour necrosis factor alpha. Annals of the rheumatic diseases. 2000; 59 Suppl 1: i75-80.

48. Wegner $\mathrm{N}$, Lundberg $\mathrm{K}$, Kinloch $\mathrm{A}$, et al. Autoimmunity to specific citrullinated proteins gives the first clues to the etiology of rheumatoid arthritis. Immunological reviews. 2010; 233: 34-54.

49. Tan YK and Conaghan PG. Imaging in rheumatoid arthritis. Best practice \& research Clinical rheumatology. 2011; 25: 569-84.

50. Monaco C, Nanchahal J, Taylor P and Feldmann M. Anti-TNF therapy: past, present and future. International immunology. 2015; 27: 55-62.

51. Burmester GR and Pope JE. Novel treatment strategies in rheumatoid arthritis. Lancet. 2017; 389: $2338-48$

52. Walitt B, Pettinger M, Weinstein A, et al. Effects of postmenopausal hormone therapy on rheumatoid arthritis: the women's health initiative randomized controlled trials. Arthritis and rheumatism. 2008; 59: 302-10.

53. Holroyd CR and Edwards CJ. The effects of hormone replacement therapy on autoimmune disease: rheumatoid arthritis and systemic lupus erythematosus. Climacteric : the journal of the International Menopause Society. 2009; 12: 378-86. 
54. Lugo L, Villalvilla A, Largo R, Herrero-Beaumont $G$ and Roman-Blas JA. Selective estrogen receptor modulators (SERMs): new alternatives for osteoarthritis? Maturitas. 2014; 77: 380-4.

55. da Silva JA, Colville-Nash P, Spector TD, Scott DL and Willoughby DA. Inflammation-induced cartilage degradation in female rodents. Protective role of sex hormones. Arthritis and rheumatism. 1993; 36: 1007-13.

56. Kavas A, Cagatay ST, Banerjee S, Keskin D and Tezcaner A. Potential of Raloxifene in reversing osteoarthritis-like alterations in rat chondrocytes: an in vitro model study. Journal of biosciences. 2013; 38: 135-47.

57. Turner AS, Athanasiou KA, Zhu CF, Alvis MR and Bryant HU. Biochemical effects of estrogen on articular cartilage in ovariectomized sheep. Osteoarthritis and cartilage / OARS, Osteoarthritis Research Society. 1997; 5: 63-9.

58. Wluka AE, Davis SR, Bailey M, Stuckey SL and Cicuttini FM. Users of oestrogen replacement therapy have more knee cartilage than non-users. Annals of the rheumatic diseases. 2001; 60: 332-6. 59. Sniekers $\mathrm{YH}$, Weinans $\mathrm{H}$, Bierma-Zeinstra SM, van Leeuwen JP and van Osch GJ. Animal models for osteoarthritis: the effect of ovariectomy and estrogen treatment - a systematic approach. Osteoarthritis and cartilage / OARS, Osteoarthritis Research Society. 2008; 16: 533-41.

60. Christgau S, Tanko LB, Cloos PA, et al. Suppression of elevated cartilage turnover in postmenopausal women and in ovariectomized rats by estrogen and a selective estrogen-receptor modulator (SERM). Menopause. 2004; 11: 508-18.

61. Karsdal MA, Bay-Jensen $A C$, Henriksen $K$ and Christiansen $C$. The pathogenesis of osteoarthritis involves bone, cartilage and synovial inflammation: may estrogen be a magic bullet? Menopause international. 2012; 18: 139-46.

62. Karsdal MA, Bay-Jensen AC, Lories RJ, et al. The coupling of bone and cartilage turnover in osteoarthritis: opportunities for bone antiresorptives and anabolics as potential treatments? Annals of the rheumatic diseases. 2014; 73: 336-48.

63. Carbone LD, Nevitt MC, Wildy K, et al. The relationship of antiresorptive drug use to structural findings and symptoms of knee osteoarthritis. Arthritis and rheumatism. 2004; 50: 351625.

64. Ham KD and Carlson CS. Effects of estrogen replacement therapy on bone turnover in subchondral bone and epiphyseal metaphyseal cancellous bone of ovariectomized cynomolgus monkeys. Journal of bone and mineral research : the official journal of the American Society for Bone and Mineral Research. 2004; 19: 823-9. 
65. Yoshida A, Morihara T, Matsuda K, et al. Immunohistochemical analysis of the effects of estrogen on intraarticular neurogenic inflammation in a rat anterior cruciate ligament transection model of osteoarthritis. Connective tissue research. 2012; 53: 197-206.

66. Dawson-Basoa ME and Gintzler AR. Estrogen and progesterone activate spinal kappa-opiate receptor analgesic mechanisms. Pain. 1996; 64: 608-15.

67. Smith YR, Stohler CS, Nichols TE, Bueller JA, Koeppe RA and Zubieta JK. Pronociceptive and antinociceptive effects of estradiol through endogenous opioid neurotransmission in women. The Journal of neuroscience : the official journal of the Society for Neuroscience. 2006; 26: 5777-85.

68. Vincent K, Warnaby C, Stagg CJ, Moore J, Kennedy S and Tracey I. Brain imaging reveals that engagement of descending inhibitory pain pathways in healthy women in a low endogenous estradiol state varies with testosterone. Pain. 2013; 154: 515-24. 
